\title{
Autosomal Dominant Polycystic Kidney Disease with Liver and Pancreatic Involvement
}

\author{
Soumya Jain ${ }^{1}$, Suresh V. Phatak ${ }^{2}$, Amruta Varma ${ }^{3}$, Rajasbala P. Dhande ${ }^{4}$ \\ ${ }^{1}$ Department of Radiodiagnosis, JNMC, Wardha, Maharashtra, India. ${ }^{2}$ Department of \\ Radiodiagnosis, JNMC, Wardha, Maharashtra, India. ${ }^{3}$ Department of Radiodiagnosis, JNMC, \\ Wardha, Maharashtra, India. ${ }^{4}$ Department of Radiodiagnosis, JNMC, Wardha, Maharashtra, India.
}

Autosomal dominant polycystic kidney disease (adult polycystic kidney disease, Potter Type III disease) is the fourth-most common cause of end-stage renal disease.(1) Polycystic Liver Disease, the most common extrarenal manifestations of Polycystic Kidney Disease is seen in $75-90 \%$ of cases, characterized by multiple biliary cystic lesions localized in over $50 \%$ of the hepatic parenchyma. In Polycystic kidney disease, hepatic cysts develop later than the renal cysts.(2) It is associated with hypertension in about $70 \%$ of cases, cyst in pancreas in $9 \%$, saccular berry aneurysm of cerebral arteries in 3-13\%, and mitral valve prolapse.

They present with hypertension and renal enlargement. Complications include cyst haemorrhage, infection, renal calculi and rarely torsion, or rupture(2) (leading to hematuria) and chronic renal failure (which develops in $50 \%$ of patients)(1). Other complications of mass effect are vena cava compression and lower portal vein and bile duct compression that presents itself as obstructive jaundice.(2)

Imaging studies (sonography, computed tomography, magnetic resonance imaging) play an important role in the diagnosis of the disease, monitoring of progression and the detection of complications.(3) Ultrasound is an important screening tool for Polycystic Kidney Disease. One can recommend contrast-enhanced computed tomography or MRI when the Ultrasound results are equivocal or if there is need to exclude complications of the disease (e.g., renal cell carcinoma) or other differential diagnosis (e.g. tuberous sclerosis).(1) Imaging is also helpful in detecting extrarenal manifestations of Polycystic Kidney Disease, most significant being intracranial aneurysms and cystic liver diseases.(3) Ultrasound can also be used to visualize cysts in other abdominal organs.

On Ultrasound, simple renal cysts appear anechoic with sharply defined imperceptible walls with posterior acoustic enhancement and lateral shadowing. Echogenic material within the cyst with no internal blood flow is seen in cases of cysts with hemorrhage or infection. Calcifications may develop. Renal cell carcinomas in contrast, although usually cystic in the setting of Autosomal Dominant Polycystic Kidney Disease, will have solid components of thick septa with blood flow. Sometimes perinephric hematomas and collections of variable echogenicity surrounding the kidney may also be seen.

CT is very sensitive in diagnosing and excellent at characterizing renal cysts. Simple renal cysts appear as thin walled rounded structures with near water attenuation $(\sim 0 \mathrm{HU})$. Cysts with internal complications may be hyperattenuating with internal non-enhancing septations and/or calcifications. On MRI, renal cysts appear morphologically the same as on CT, well-defined rounded structures with very thin regular walls.

\author{
Corresponding Author: \\ Dr. Suresh V. Phatak, \\ Department of Radiodiagnosis, \\ JNMC, Sawangi, Meghe, wardha-442001, \\ Maharashtra, India. \\ E-mail: suresh_phatak@yahoo.com
}

DOI: $10.14260 / \mathrm{jemds} / 2020 / 356$

Financial or Other Competing Interests: None.

How to Cite This Article: Jain S, Phatak SV, Varma A, et al. Autosomal dominant polycystic kidney disease with liver and pancreatic involvement. J. Evolution Med. Dent. Sci. 2020;9(20):16251626, DOI: 10.14260/jemds/2020/356

Submission 21-01-2020,

Peer Review 20-03-2020,

Acceptance 27-03-2020,

Published 18-05-2020.

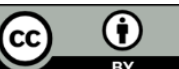


Autosomal Dominant Polycystic Kidney Disease is a multifactorial disorder characterized by bilateral renal cysts usually affecting the adult patients. Liver cysts are the most common extrarenal manifestation and are often incidental findings and clinically insignificant. Ultrasound is a useful screening tool for polycystic kidney disease in adolescents.

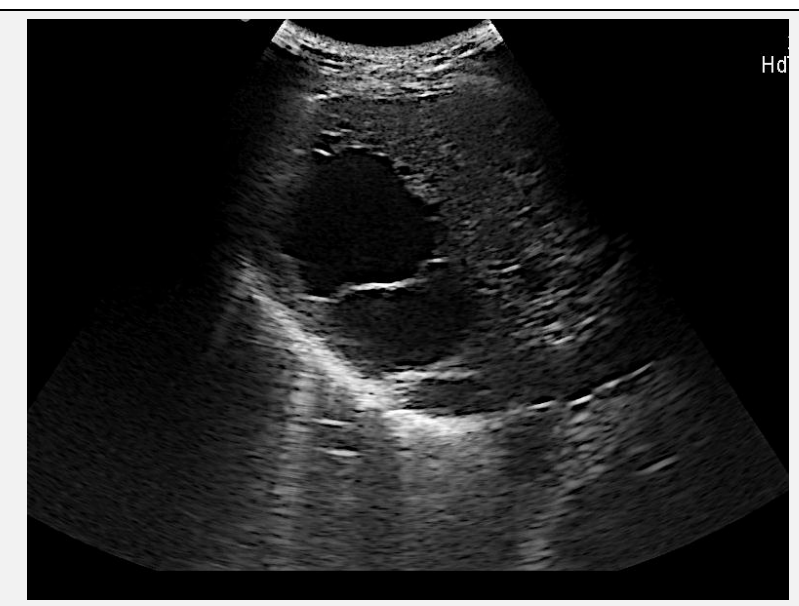

Transabdominal Ultrasound Image Showing Multiple Liver Cysts
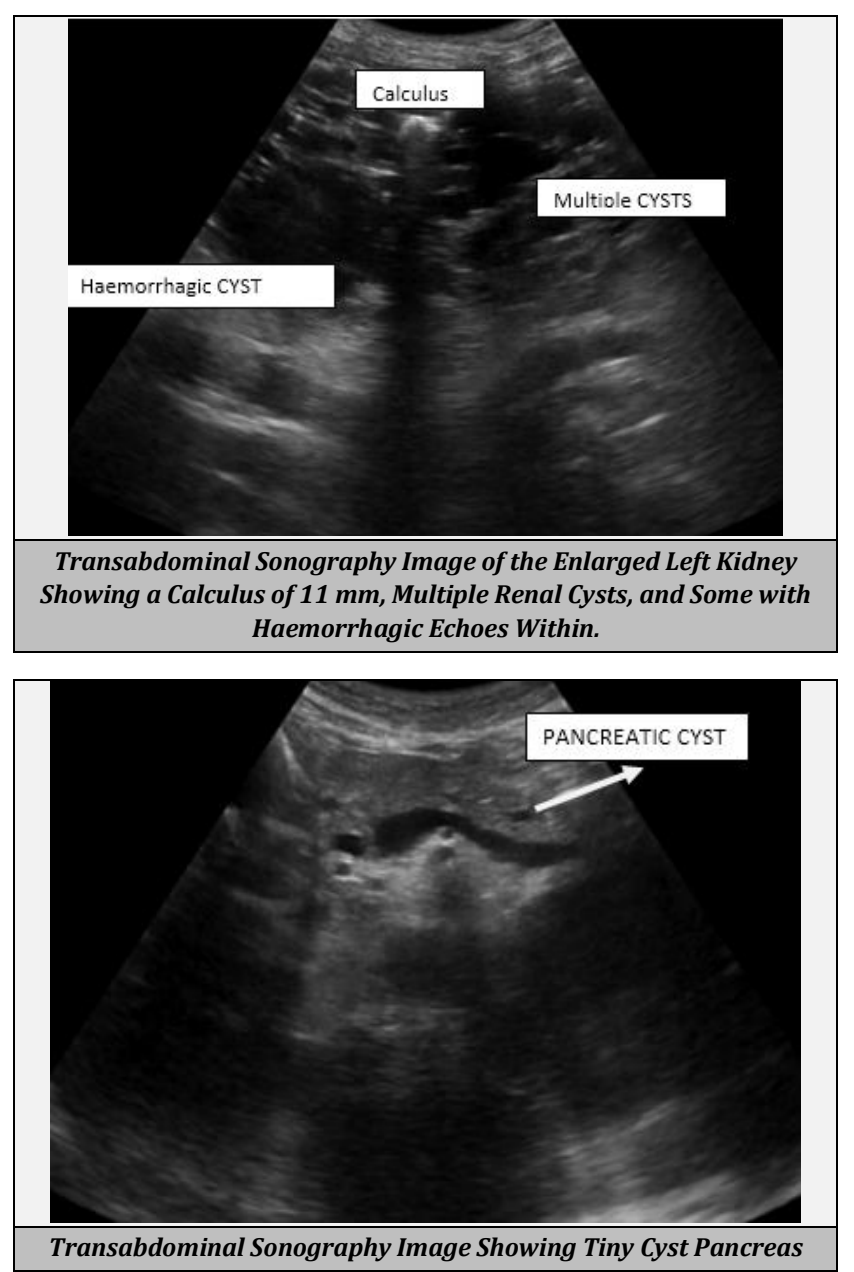

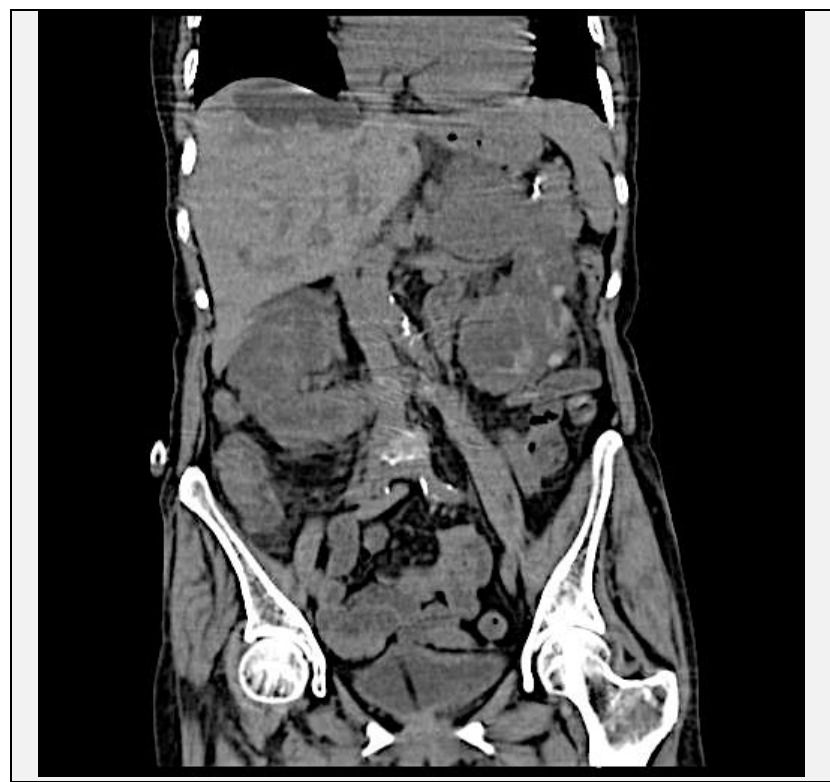

Coronal Non-Contrast CT Scan Showing Multiple Cystic Lesions in Both Kidneys Causing Compression and Distortion of the Collecting System with Marked Thinning of the Renal Cortex with Blood Density Collection Noted in Multiple Cysts Bilaterally and Similar Cystic Lesions were seen in Both Right and Left Lobes of Liver

\section{REFERENCES}

[1] Ekechukwu KU, Hecht A, Nwakudu OA. Autosomal dominant polycystic disease (ADPD). Applied Radiology 2013;42(5):29-30.

[2] De Miranda HMS, De Morais VEJ. The liver and polycystic kidney disease. Chap -17. In: Li X, edr. Polycystic Kidney Disease. Brisbane, AU: Codon Publications 2015.

[3] Gradzik M, Niemczyk M, Gołębiowski M, et al. Diagnostic imaging of autosomal dominant polycystic kidney disease. Polish Journal of Radiology 2016;81:441-53. 